\title{
Formation of volatile compounds, peptidolysis and carbohydrate fermentation by mesophilic lactobacilli and streptoccocci cultures in a cheese extract
}

\author{
Guillermo Hugo Peralta ${ }^{1}$ - I. V. Wolf ${ }^{1}$. \\ M. C. Perotti ${ }^{1,2}$ - C. V. Bergamini ${ }^{1}$ E. R. Hynes ${ }^{1,2}$
}

Received: 11 January 2016 /Revised: 27 March 2016/Accepted: 28 April 2016 /

Published online: 12 May 2016

(C) INRA and Springer-Verlag France 2016

\begin{abstract}
Many studies about the influence of Lactococcus lactis on cheese flavour have been reported, while the impact of mesophilic lactobacilli and Streptococcus thermophilus, either as single or mixed cultures, have been less studied. The ability of Lactobacillus paracasei 90, Lb. casei 72 (INLAIN collection) and S. thermophilus 2 (commercial strain) to produce flavour-related biochemical changes, as single or mixed (lactobacilli + streptococci) cultures, was assessed in a cheese model. These three strains, with different activities of glutamate dehydrogenase (GDH) and aminotransferases (AT), were incubated $\left(14 \mathrm{~d} / 37^{\circ} \mathrm{C}\right)$ alone or in mixed cultures in a cheese model consisting of a sterile extract of a fresh cheese. Mesophilic lactobacilli showed fermentation of carbohydrates, an increase of peptidolysis and production of volatile compounds that were correlated with their AT activities. In addition, these strains also produced completely different profiles of the amino acceptor compounds: pyruvate and $\alpha$-ketoglutarate. S. thermophilus 2 increased the level of $\alpha$-ketoglutarate due to its high GDH activity, but its contribution to flavour compound production was negligible; small additional changes existed when $S$. thermophilus 2 was mixed with the lactobacilli. Biochemical changes leading to flavour formation were mainly due to the activity of lactobacilli. Our results suggest that the AT profile and the peptidolytic activity of each strain influenced the volatilome of the extracts during incubation. So, both lactobacilli strains could be used as adjunct cultures in cheeses to increase/ diversify the flavour, but more studies are needed to deepen the knowledge about the potential of $S$. thermophilus 2 for the production of flavour compounds.
\end{abstract}

Guillermo Hugo Peralta

gperalta@fiq.unl.edu.ar

1 Instituto de Lactología Industrial, Universidad Nacional del Litoral — Consejo Nacional de Investigaciones Científicas y Técnicas, Santiago del Estero 2829, S3000AOM Santa Fe, Argentina

2 Facultad de Ingeniería Química, Universidad Nacional del Litoral, Santiago del Estero 2829, S3000AOM Santa Fe, Argentina 
Keywords Streptococci - Mesophilic lactobacilli · Volatile compounds · Cheese extract . Cheese flavour

\section{Introduction}

Cheese flavour formation is a dynamic and complex process in which several metabolic pathways are interrelated. In particular, amino acid (AA) catabolism by lactic acid bacteria is one of the most important biochemical events that leads to production of the most important flavour compounds (Yvon 2006; Steele et al. 2013). The AA catabolism by lactic acid bacteria (LAB) begins mainly by a transamination reaction catalysed by aminotransferases (AT), in which the amino group of an AA is transferred to a suitable acceptor, usually $\alpha$-ketoglutarate. This compound is present at low levels in cheese and has been identified as the main limiting factor for transamination reactions (Steele et al. 2013). However, it can be replenished by oxidative deamination from glutamic acid, which is often present at high levels in cheeses during ripening by a reaction catalysed by the enzyme glutamate dehydrogenase (GDH) (Tanous et al. 2002; Yvon et al. 1998). In this way, the use of lactobacilli or lactococci with high levels of GDH activity has led to an increase of flavour development via AA catabolism (De Angelis et al. 2010; Williams et al. 2006). A similar effect was observed when a Lactobacillus strain $\mathrm{GDH}+$ was assayed in a matrix with the addition of glutamic acid (Kieronczyk et al. 2003). In addition, the incorporation of $\alpha$-ketoglutarate in cheeses, when an adjunct culture of $L b$. plantarum with low GDH activity was used, increased AA catabolism and the subsequent production of flavour compounds (De Angelis et al. 2010). This approach was also assayed in Saint Paulin and Cheddar cheeses in which an intensification of aroma was observed (Banks et al. 2001; Yvon et al. 1998). Conversely, Kieronczyk et al. (2004) demonstrated that not only the quantity but also the nature of volatile compounds in cheese depends on the AT and, in this case, on their profile in a given strain. In this sense, it has been proposed that competition exists between AT enzymes for the amino group acceptor available in the cheese (Kieronczyk et al. 2004; Thage et al. 2005). In addition to $\alpha$-ketoglutarate, other $\alpha$ ketoacids, such as pyruvate or oxaloacetate, were demonstrated to be efficient amino group acceptors for some ATs (Amárita et al. 2001; Tanous et al. 2005).

The selection of LAB that possess key enzymatic activities (AT and GDH) and their use in mixed cultures with complementary enzymatic profiles have been proposed as an approach to increase flavour in cheese (Gutiérrez-Méndez et al. 2008; Smit et al. 2009). In particular, interest has been paid to elucidate whether cooperation between strains showing either high GDH or AT activities was possible. In this way, Kieronczyk et al. (2003) reported cooperation between strains of Lactobacillus GDH+ (Lb. casei 2576 and Lb. paracasei subsp. paracasei INF15D), which produced $\alpha$-ketoacids from different AAs, and a strain of Lactococcus lactis GDH- (L. lactis subsp. cremoris NCDO763), which metabolised these compounds with the production of carboxylic acids. This led to a global increase of flavour, in an in vitro experiment. Ayad et al. (2001) also verified cooperation between two strains of Lactococcus lactis: one with high peptidolytic ability and the other with AT and $\alpha$-ketoacid decarboxylase activities. In particular, the production of 3- 
methylbutanal increased when these two strains were added together, which the authors attributed to the complementary events of peptidolysis and AA catabolism in an experiment conducted in milk.

Streptococcus thermophilus is the second most used lactic acid bacterial species in the dairy industry. It is employed as a starter culture for yogurt, many cheese varieties and other dairy fermented foods. In Argentina, $S$. thermophilus starters are the main cultures within the cheese industry, as most important cheeses in terms of volume of production are produced with this thermophilic starter. Despite its enormous economic and technological importance, the influence of $S$. thermophilus on cheese flavour is not well known. Although $S$. thermophilus is considered to be a strong acid producer species, though a poor flavour producer, few studies have characterised the profile of volatile compounds in cheeses made with strains of this species (Bergamini et al. 2010; Milesi et al 2010; Wang et al. 2012). In relation to the production of flavour compounds via AA catabolism, several strains of $S$. thermophilus have shown high levels of GDH and AT activities (Helinck et al 2004; Yvon 2006). Concerning the carbohydrate fermentation, most strains of $S$. thermophilus are not able to ferment galactose after lactose depletion, which causes its accumulation in cheeses when it is used as the single starter culture (McSweeney and Sousa 2000). The high levels of residual galactose may favour the growth of NSLAB, which could lead to defects such as unwanted eyes, off-flavour or browning (Porcellato et al. 2015).

In the present work, we assessed the ability of three strains (two mesophilic lactobacilli: Lb. paracasei 90 and Lb. casei 72, and one streptococcus: $S$. thermophilus 2 ) to produce flavour-related biochemical changes as single cultures in a cheese extract and monitored their ability to cooperate for this activity when inoculated as a mixed culture (lactobacilli + streptococci). These strains were previously characterised in relation to their GDH and AT activities. $S$. thermophilus 2 showed a GDH NAD-dependent activity which was ten times as high as that observed in both lactobacilli strains. As for AT activities, lactobacilli showed different profiles of specificity: Lb. casei 72 had similar activity towards Asp, Met, branched-chain (Leu, Ile, Val) and aromatic (Phe, Tyr, Trp) amino acids, while in Lb. paracasei 90 AT activity towards Asp was three times higher than towards the rest of the tested amino acids. AT activities of $S$. thermophilus 2 were slightly higher towards Asp, Leu and Tyr than to the other studied amino acids (Peralta et al. 2016).

\section{Material and methods}

\subsection{Strains and culture conditions}

In the present work, we studied two strains of mesophilic lactobacilli: Lb. paracasei 90 and $L b$. casei 72, and one strain of streptococci: S. thermophilus 2. Both strains of lactobacilli were isolated from good quality cheese, and they belong to the collection of the Instituto de Lactología Industrial (INLAIN), while the S. thermophilus 2 is a commercial strain.

Stock cultures of the selected strains were stored at $-80{ }^{\circ} \mathrm{C}$ in MRS broth (Biokar Diagnostics, Beauvais, France) for lactobacilli and Elliker broth (Biokar Diagnostics, 
Beauvais, France) for streptococci, with the addition of glycerol $15 \%(v / v)$ as a cryopreservative. Before use, each strain was cultivated twice in MRS and Elliker broth at $37{ }^{\circ} \mathrm{C}$ overnight for lactobacilli and streptococci, respectively.

\subsection{Cheese extract and experimental design}

The abilities of the strains to produce biochemical changes related to the production of flavour in cheese were studied in a cheese model consisting of a sterile extract of soft cheese. To prepare the extract, a batch of Cremoso cheese was made especially in our pilot plant using the same starter of $S$. thermophilus 2 and no adjunct culture. Cheesemaking was performed according to Milesi et al. (2009). Cremoso cheese is a high fat and high moisture cheese, with a minimum of $50 \%$ fat in the dry matter and a moisture content ranging between 46 and $55 \%$.

After 20 days of ripening, a representative portion of Cremoso cheese was disintegrated and homogenised with distilled water (1:1), and the resultant slurry was twice centrifuged $\left(17,000 \mathrm{xg}-15 \mathrm{~min}-10{ }^{\circ} \mathrm{C}\right)$. The soluble fraction was filtered through glass wool (AP 25 EM, Millipore, Sao Paolo, Brazil), and the $\mathrm{pH}$ value and salt content were measured and adjusted (if necessary) to 5.20 and $1.5 \%(\mathrm{w} / \mathrm{v})$, respectively. Finally, the extract was heat treated at $70{ }^{\circ} \mathrm{C}$ for $30 \mathrm{~min}$ and filtered through 0.45- $\mu \mathrm{m}$ PVDF membranes (Millipore, Sao Paulo, Brazil).

A volume of the extract $(70 \mathrm{~mL})$ was inoculated with the single strains S. thermophilus 2 (St), Lb. paracasei 90 (90) and Lb. casei 72 (72) and with combinations of $S$. thermophilus 2 and mesophilic lactobacilli ( $\mathrm{St}+90$ and $\mathrm{St}+72)$ at a level of $7 \times 10^{5}$ CFU.mL ${ }^{-1}$ each. Aliquots $(15 \mathrm{~mL})$ were distributed in screwcap tubes and incubated at $37{ }^{\circ} \mathrm{C}$ for 14 days. In parallel, control non-inoculated extracts were incubated. After that, the tubes were maintained at $-18{ }^{\circ} \mathrm{C}$ until analysis. All of the experiments were performed in duplicate using two independent cultures of each strain for inoculation.

\subsection{Microbiological counts}

Viable counts of mesophilic lactobacilli and streptococci in extracts were recorded at $0,3,7$ and 14 days of incubation by plating on MRS and Elliker (Biokar Diagnostics, Beauvais, France) agar, respectively, and incubating at $37{ }^{\circ} \mathrm{C}$ for 48 and $24 \mathrm{~h}$, respectively. In MRS agar, the lactobacilli strains only grew. On the contrary, both lactobacilli and streptococci grew on Elliker, but the differential count was made based in different aspects of colonies and times of incubation; streptococci colonies were larger and whiter than that of the lactobacilli colonies and grew at $24 \mathrm{~h}$ of incubation while lactobacilli colonies could be counted after $48 \mathrm{~h}$ of incubation. Sterility in control extracts was checked by plating samples on skim-milk agar and incubating at $37^{\circ} \mathrm{C}$ for $48 \mathrm{~h}$.

\subsection{Organic acids and carbohydrates}

The concentration of eight organic acids (citric, $\alpha$-ketoglutaric, pyruvic, lactic and acetic acid) and two carbohydrates (lactose and galactose) was quantified in the final cheese extracts after 3 and 14 days of incubation. The analyses were 
performed by using an HPLC with an Aminex HPX-87H column $(300 \times 7.8 \mathrm{~mm})$ equipped with a cation $\mathrm{H}+$ microguard cartridge (Bio-Rad Laboratories, USA), which allows the simultaneous quantification of sugars and organic acids using UV and IR detectors connected in series. Chromatographic separation of most compounds was carried out isocratically at $65{ }^{\circ} \mathrm{C}$ with a mobile phase of $10 \mathrm{mM}$ $\mathrm{H} 2 \mathrm{SO} 4$ at a flow rate of $0.6 \mathrm{~mL} \cdot \mathrm{min}^{-1}$. For the best resolution and quantification of $\alpha$-ketoglutaric, chromatographic separation of this compound was carried out at different temperatures and concentrations of the mobile phase, $32{ }^{\circ} \mathrm{C}$ and $16 \mathrm{mM}$ $\mathrm{H} 2 \mathrm{SO} 4$, respectively. HPLC equipment consisted of a quaternary pump, an online degasser, a column oven, a UV-visible detector (all Series 200) and a refractive index detector thermostated at $35^{\circ} \mathrm{C}$ (Series Flexar) (Perkin Elmer, USA). The UV detector was set at $210 \mathrm{~nm}$ for the detection of organic acids, while the IR detector was used for the analysis of carbohydrates. Data were collected and processed on a computer with Chromera ${ }^{\circledR}$ software (Perkin Elmer). Analytical grade carbohydrates and organic acids (Sigma Aldrich, USA) were used as standards to obtain calibration curves, which were used for the quantification.

\subsection{Free amino acids}

FAAs in extracts after 14 days of incubation were analysed by HPLC after derivatisation using the chemistry package of the Waters AccQ-Tag Amino Analysis Method (Waters Corp., Milford, MA) according to Bergamini et al. (2010). The HPLC equipment was the same as that used for organic acid and carbohydrate analysis.

\subsection{Analysis of volatile compounds by SPME-GC-FID/MS}

Volatile compounds in extracts at 3 and 14 days of incubation were analysed by SPME-GC-FID/MS according to Peralta et al. (2014). For SPME, a CAR/PDMS 75- $\mu \mathrm{m}$ (Supelco Inc. Bellefonte, PA, USA) fibre was used, which was exposed for $30 \mathrm{~min}$ into the headspace of vials containing $15 \mathrm{~mL}$ of each extract that was previously thermostated at $40{ }^{\circ} \mathrm{C}$ for $10 \mathrm{~min}$. The volatile compounds retained on the fibre coating phase were thermally desorbed in the injection port $\left(250{ }^{\circ} \mathrm{C}\right.$, splitless mode) equipped with a narrow-bore glass liner (Supelco, Bellefonte, USA) of a gas chromatograph (Perkin Elmer model 9000, USA). The compounds were separated on an HP Innowax column $(60 \mathrm{~m} \times 0.25 \mathrm{~mm} \times 0.25 \mu \mathrm{m})$ (Agilent J\&W, Agilent Technologies, USA).

\subsection{Statistical analysis}

The data measuring $\mathrm{pH}$, microbial counts, carbohydrates, organic acids, amino acids and volatile compounds in control and experimental cheese extracts were subjected to one-way analysis of variance (ANOVA) using SPSS 10.0 software (SPSS Inc., Chicago, USA). When differences were found, means of $\mathrm{pH}$, microbial counts and total FAA were compared by the Bonferroni test using the same tool. Finally, principal component analysis (PCA) was applied to results of volatile compounds using the same software. 


\section{Results}

\subsection{Cell counts and pH}

Control extracts remained sterile during incubation. The initial counts of S. thermophilus 2 were $7.3 \times 10^{5}, 5.7 \times 10^{5}$ and $9 \times 10^{5}$ CFU.mL ${ }^{-1}$ in extracts St, St+ 90 and St+72, respectively. After 3 days of incubation, $S$. thermophilus 2 in the St extracts had diminished to $1.8 \times 10^{4} \mathrm{CFU} . \mathrm{mL}^{-1}$, while no viable cells were detected at 7 and 14 days of incubation. In St+90 and St+72, S. thermophilus 2 was not detected at any time during incubation, while the counts of Lb. paracasei 90 and Lb. casei 72 showed an increase at day 3 (3 to $\left.8 \times 10^{8} \mathrm{CFU} \cdot \mathrm{mL}^{-1}\right)$ and then a decrease of approximately 2 to $3 \mathrm{log}$. At day 7, the decrease was more marked for Lb. casei 72 . The counts of lactobacilli at the end of incubation did not significantly differ between extracts $(p>0.05)$; however, the numeric values were slightly higher in extracts in which these strains were inoculated alone in comparison to when they were inoculated together with $S$. thermophilus 2, above all for Lb. casei 72 (Table 1).

The $\mathrm{pH}$ remained at the initial target value of 5.20 until the end of incubation in $\mathrm{C}$ and St extracts. On the contrary, in extracts inoculated with lactobacilli, the $\mathrm{pH}$ decreased to 4.20-4.30 on day 3 of incubation. After that, the $\mathrm{pH}$ diminished more slowly, reaching final values of 3.90-4.00 at 14 days of incubation; the values were lower for extracts with Lb. casei 72 (Table 2).

\subsection{Carbohydrates and organic acids}

The initial concentration of lactose and galactose in all extracts was ca. 45 and $554 \mathrm{mg} /$ $100 \mathrm{~mL}$, respectively; it decreased during incubation in extracts with lactobacilli but remained constant when only $S$. thermophilus was inoculated. In effect, significantly lower levels $(p \leq 0.05)$ of these carbohydrates were observed in all extracts inoculated with lactobacilli, especially in extracts 72 and St+72, when compared with $\mathrm{C}$ and $\mathrm{St}$ extracts both at 3 and 14 days of incubation (Table 3).

Table 1 Lactobacilli counts on MRS agar $\left(\log \mathrm{CFU} \cdot \mathrm{mL}^{-1}\right)$ in extracts during incubation at $37^{\circ} \mathrm{C}$

\begin{tabular}{|c|c|c|c|c|}
\hline \multirow[t]{2}{*}{ Extracts } & \multicolumn{4}{|c|}{ Incubation time (days) } \\
\hline & 0 & 3 & 7 & 14 \\
\hline 90 & $5.50 \pm 0.54$ & $8.91 \pm 0.01 b$ & $7.21 \pm 0.12 b$ & $5.93 \pm 0.28$ \\
\hline $\mathrm{St}+90$ & $5.48 \pm 0.62$ & $8.86 \pm 0.05 b$ & $7.25 \pm 0.10 \mathrm{~b}$ & $5.61 \pm 0.49$ \\
\hline 72 & $5.64 \pm 0.06$ & $8.52 \pm 0.01 \mathrm{a}$ & $6.04 \pm 0.06 \mathrm{a}$ & $6.25 \pm 0.08$ \\
\hline $\mathrm{St}+72$ & $5.57 \pm 0.12$ & $8.58 \pm 0.01 \mathrm{a}$ & $6.01 \pm 0.01 \mathrm{a}$ & $5.43 \pm 0.10$ \\
\hline
\end{tabular}

Mean values \pm standard deviation of two independent experiments are reported. Values with different letters within the same column are significantly different $(p \leq 0.05)$. The letters have not been included in the cases when significant differences were not found $(p>0.05)$

90 extracts inoculated with $\mathrm{Lb}$. paracasei $90, \mathrm{St}+90$ extracts inoculated with $\mathrm{Lb}$. paracasei 90 and S. thermophilus 2, 72 extracts inoculated with $\mathrm{Lb}$. casei 72, St+72: extracts inoculated with $\mathrm{Lb}$. casei 72 and $S$. thermophilus 2 
Table 2 Evolution of $\mathrm{pH}$ values in extracts during incubation at $37^{\circ} \mathrm{C}$

\begin{tabular}{|c|c|c|c|c|}
\hline \multirow[t]{2}{*}{ Extracts } & \multicolumn{4}{|c|}{ Incubation time (days) } \\
\hline & 0 & 3 & 7 & 14 \\
\hline $\mathrm{C}$ & $5.20 \pm 0.01$ & $5.20 \pm 0.01 \mathrm{c}$ & $5.25 \pm 0.01 b$ & $5.15 \pm 0.01 \mathrm{c}$ \\
\hline St & $5.20 \pm 0.01$ & $5.19 \pm 0.01 \mathrm{c}$ & $5.25 \pm 0.01 \mathrm{~b}$ & $5.15 \pm 0.01 \mathrm{c}$ \\
\hline 90 & $5.20 \pm 0.01$ & $4.20 \pm 0.01 \mathrm{a}$ & $4.05 \pm 0.01 \mathrm{a}$ & $4.00 \pm 0.01 \mathrm{~b}$ \\
\hline $\mathrm{St}+90$ & $5.20 \pm 0.01$ & $4.20 \pm 0.01 \mathrm{a}$ & $4.05 \pm 0.07 \mathrm{a}$ & $4.00 \pm 0.01 \mathrm{~b}$ \\
\hline 72 & $5.20 \pm 0.01$ & $4.28 \pm 0.04 \mathrm{a}, \mathrm{b}$ & $4.00 \pm 0.01 \mathrm{a}$ & $3.93 \pm 0.04 \mathrm{a}$ \\
\hline $\mathrm{St}+72$ & $5.20 \pm 0.01$ & $4.28 \pm 0.04 \mathrm{a}, \mathrm{b}$ & $4.00 \pm 0.01 \mathrm{a}$ & $3.90 \pm 0.01 \mathrm{a}$ \\
\hline
\end{tabular}

Mean values \pm standard deviation of two independent experiments are reported. Values with different letters within the same column are significantly different $(\mathrm{p} \leq 0.05)$. The letters have not been included in the cases when significant differences were not found $(p>0.05)$

$C$ control non-inoculated extracts, St extracts inoculated with $S$. thermophilus 2, 90 extracts inoculated with Lb. paracasei 90, St+90 extracts inoculated with Lb. paracasei 90 and S. thermophilus 2, 72 extracts inoculated with $L b$. casei 72, St+72 extracts inoculated with Lb. casei 72 and S. thermophilus 2

Lactic, citric and acetic acids were the main organic acids in the extracts, while pyruvic and $\alpha$-ketoglutaric acids had the lowest levels. All of these compounds showed significant differences between extracts (Table 3). The levels of citric acid after 14 days of incubation was slightly higher in extracts inoculated with the strain 72, either alone or combined with St. Concentration of lactic and acetic acids significantly increased in extracts with lactobacilli; the highest levels were found in 90 and St+90 at 3 days but then changed to 72 and $\mathrm{St}+72$ after 14 days of incubation. In relation to the results of pyruvic and $\alpha$-ketoglutaric acids, which are potential amino group acceptors in the transamination reaction, the individual concentrations and results of ANOVA are shown in Table 3. In addition, and for a better visualisation of results, we show the difference in concentration of these acids between inoculated and control extracts in Fig. 1. Pyruvic acid increased in extracts containing lactobacilli, and the increase was higher in 90 and St+90 extracts. As for $\alpha$-ketoglutaric acid, it decreased significantly in 90 and St+90 extracts but increased in St and 72 after 3 days. Its concentration was also significantly higher in St, 72 and St+72 in relation to other extracts after 14 days of incubation.

\subsection{Free amino acids}

The level of total FAAs increased during the incubation of the extracts inoculated with mesophilic lactobacilli, both when added alone and when added in combination with $S$. thermophilus 2, compared with $\mathrm{C}$ and St extracts. Total FAA levels showed significant differences $(p \leq 0.05)$ between extracts, in increasing order, as follows: $\mathrm{C}-\mathrm{St}<\mathrm{St}+90-90<72-\mathrm{St}+72$ (Fig. 2).

In addition to the total FAA concentration, the profiles of FAAs changed with the inoculums due to the selective release of some FAAs during peptidolysis. In this sense, extracts $\mathrm{C}$ and St showed similar profiles, in which Leu, Phe, Lys and Pro were the 


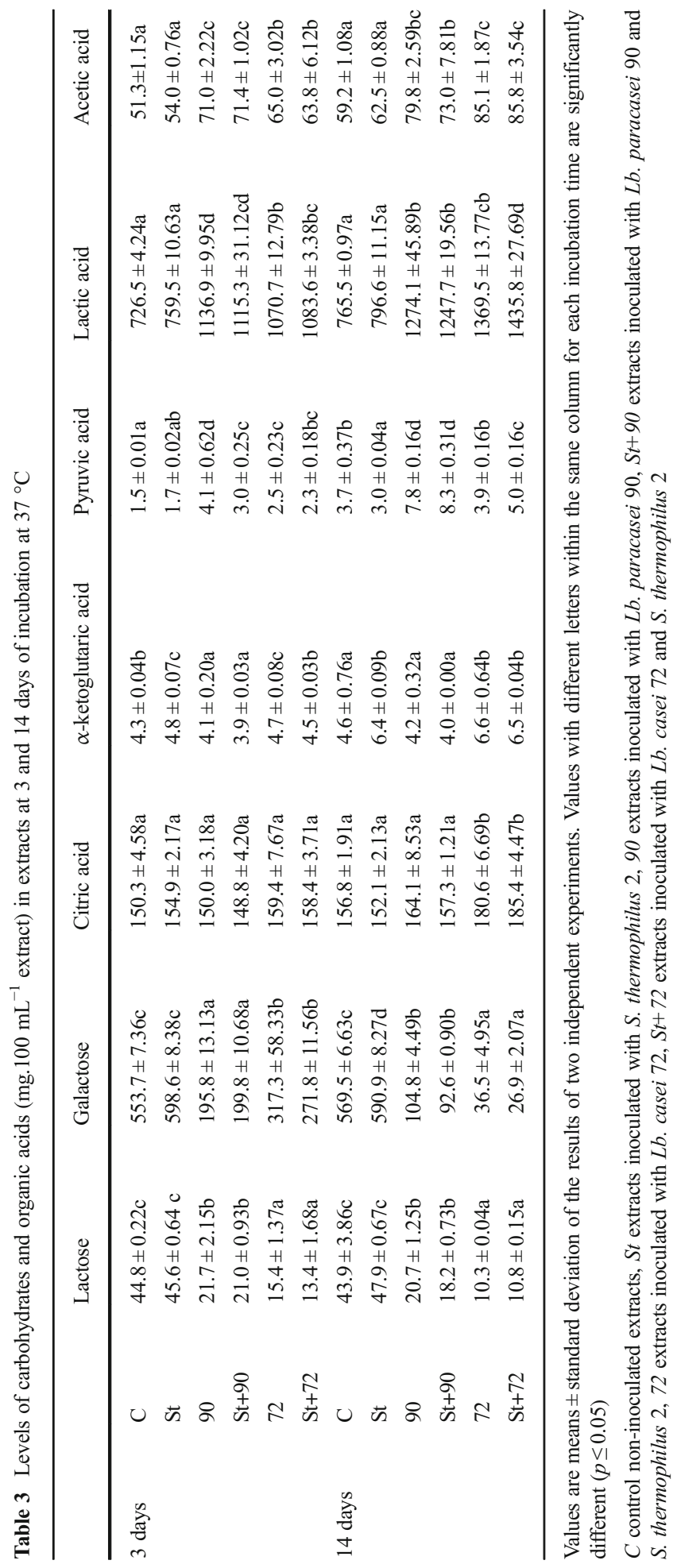




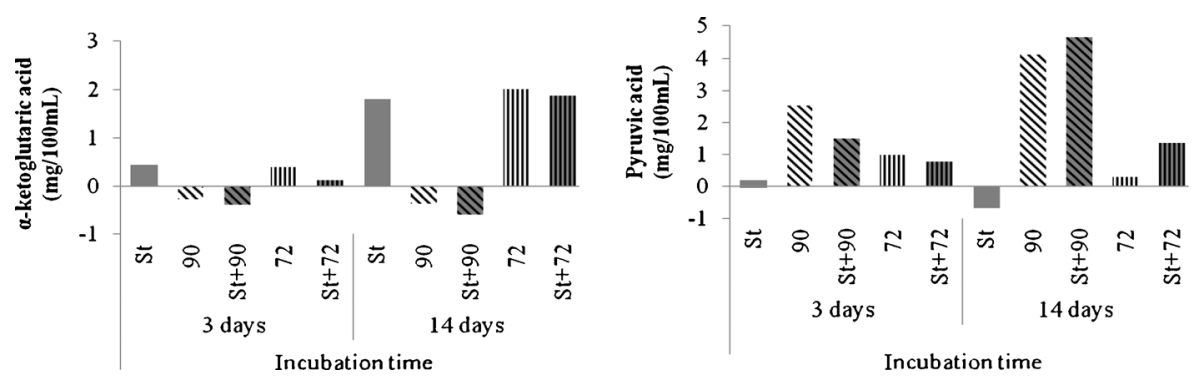

Fig. 1 Differences in the levels of $\alpha$-ketoglutaric and pyruvic acid (mg. $100 \mathrm{~mL}^{-1}$ extract) between inoculated extracts and the control extract. $C$ : control non-inoculated extracts, St: extracts inoculated with $S$. thermophilus 2, 90: extracts inoculated with $\mathrm{Lb}$. paracasei 90, St+90: extracts inoculated with Lb. paracasei 90 and S. thermophilus 2, 72: extracts inoculated with $\mathrm{Lb}$. casei 72, St+72: extracts inoculated with $\mathrm{Lb}$. casei 72 and $S$. thermophilus 2

majority FAAs, while in the extracts containing lactobacilli, the main FAAs were Leu, Glu, Phe and Arg; for 90 and St+90 extracts, the fifth main FAA was Asp (Fig. 3).

Extracts containing $L b$. casei 72 showed a significant increase $(p \leq 0.05)$ of all FAAs in relation to $\mathrm{C}$ and St extracts; overall, FAA profiles in extracts 72 and St+72 were similar, except for Leu, Val, Arg and Pro, which were significantly higher $(\mathrm{p} \leq 0.05)$ in $\mathrm{St}+72$. In the case of $\mathrm{Lb}$. paracasei 90 , both 90 and St+90 showed increased content of Asp, Glu, Leu, Phe, Pro, Val, Arg and Ser $(p \leq 0.05)$, while Ile, Tyr, Met, Lys, Gly, His, Thr and Ala remained similar to the $\mathrm{C}$ and St extracts. No difference in the level of FAAs was observed between 90 and St+90 extracts.

Thus, $S$. thermophilus 2 did not significantly increase the initial level of peptidolysis and did not cooperate with $L b$. paracasei 90 to increase peptidolysis when cocultured. On the contrary, a clue of cooperation in peptidolysis was found between Lb. casei 72 and $S$. thermophilus 2 .

\subsection{Volatile compounds}

Twenty-eight volatile compounds were identified in the extracts, including aldehydes (4), ketones, alcohols, acids and esters (Table 4). Significant $(p \leq 0.05)$ differences were

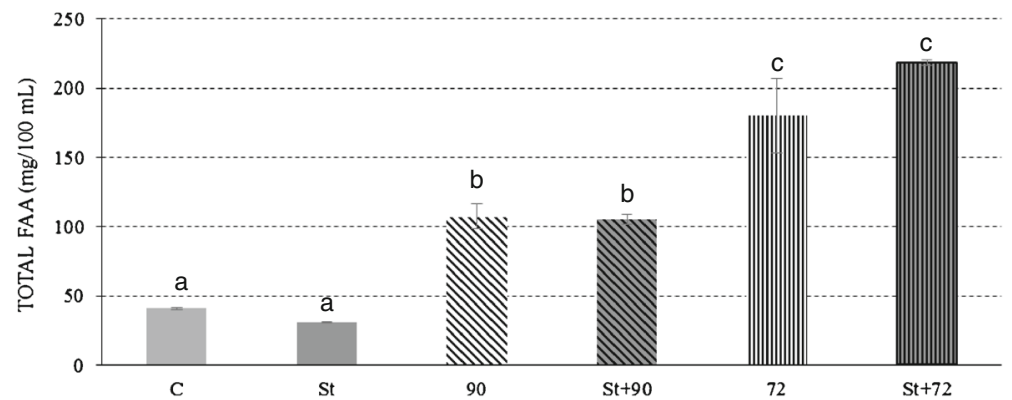

Fig. 2 Total FAAs $\left(\mathrm{mg} .100 \mathrm{~mL}^{-1}\right)$ in cheese extracts at 14 days of incubation at $37{ }^{\circ} \mathrm{C}$. Values are means \pm standard deviation of the results of two independent experiments. Bars with different letters are significantly different $(p \leq 0.05)$. $C$ : control non-inoculated extracts, St: extracts inoculated with $S$. thermophilus 2, 90: extracts inoculated with $L b$. paracasei 90, St+90: extracts inoculated with Lb. paracasei 90 and S. thermophilus 2, 72: extracts inoculated with $\mathrm{Lb}$. casei 72, St+72: extracts inoculated with $\mathrm{Lb}$. casei 72 and $S$. thermophilus 2 

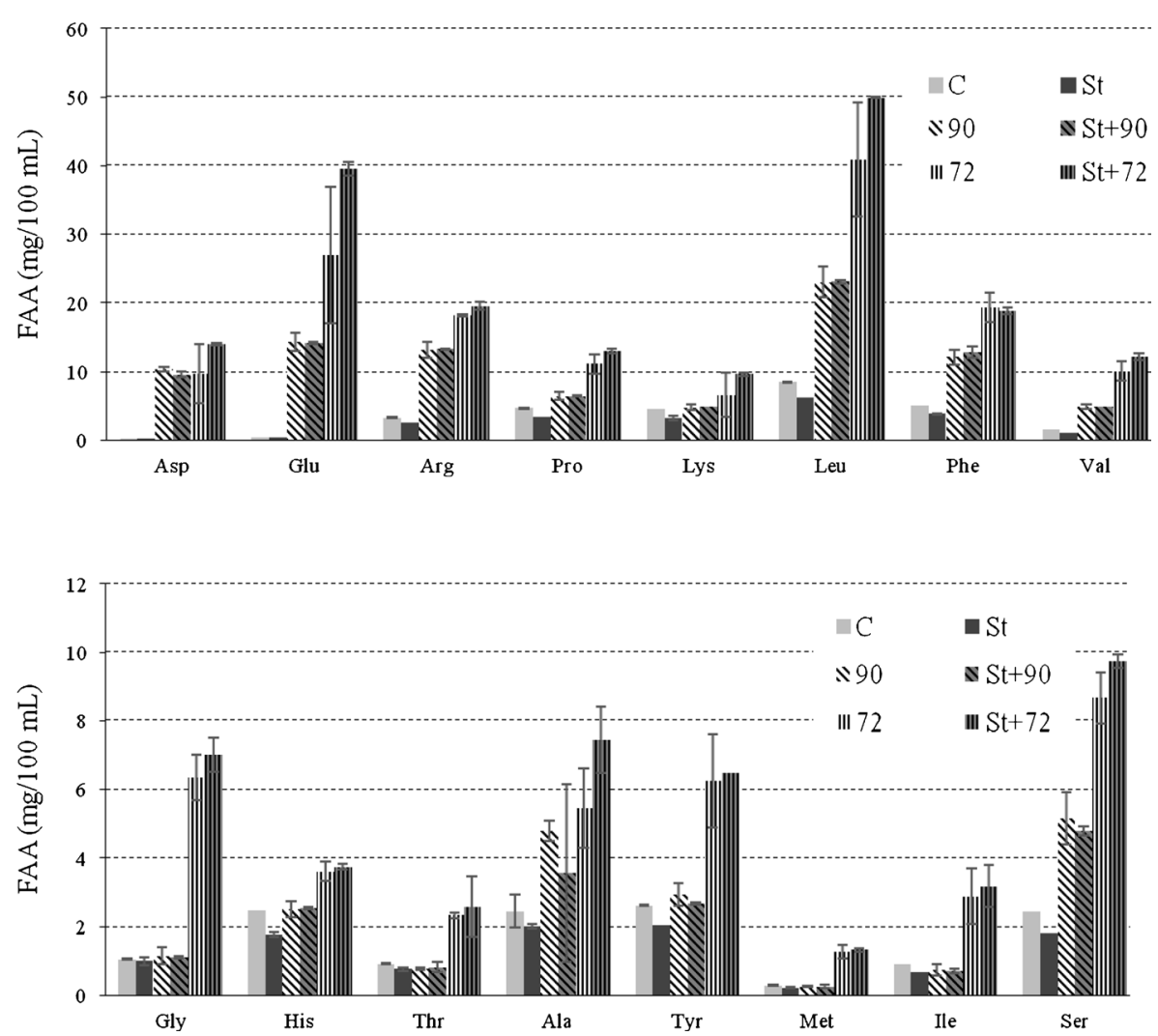

Fig. 3 Individual FAAs (mg. $100 \mathrm{~mL}^{-1}$ ) in cheese extracts at 14 days of incubation at $37{ }^{\circ} \mathrm{C}$. Values are means \pm standard deviation of the results of two independent experiments. $C$ : control non-inoculated extracts, $S t$ : extracts inoculated with $S$. thermophilus 2, 90: extracts inoculated with Lb. paracasei 90, St+90: extracts inoculated with $L b$. paracasei 90 and $S$. thermophilus 2, 72: extracts inoculated with $\mathrm{Lb}$. casei 72, St+72: extracts inoculated with Lb. casei 72 and S. thermophilus 2

detected for 15 of the volatile compounds analysed at one or at both two sampling times.

A total of nine alcohols were found, which are mentioned in Table 4. In St extracts, the level of 2-ethyl 1-hexanol increased significantly $(p \leq 0.05)$ after 3 days of incubation, while in lactobacilli-containing extracts, the levels of 1-heptanol and 1-octanol significantly increased during all incubation, especially in extracts with strain 72 . In addition, the increase of 1-heptanol for the extracts with strains 72 and 90 at 3 and 14 days, respectively, was higher when the strain of $S$. thermophilus was also inoculated. Finally, the 2-hexanol concentration increased too, in all of the extracts compared to $\mathrm{C}$ after 14 days of incubation.

As for ketones, five were detected in the extracts: 2-propanone, 2-butanone, 2hexanone, diacetyl and acetoin. The contents of ketones differed between types of extracts $(p \leq 0.05)$, except for 2-propanone. All extracts with lactobacilli showed higher levels of diacetyl at 3 days of incubation in relation to $\mathrm{C}$ and St extracts, especially those containing strain 90, which also showed increased content of acetoin. The increase of diacetyl in extracts with lactobacilli was lower when S. thermophilus 2 


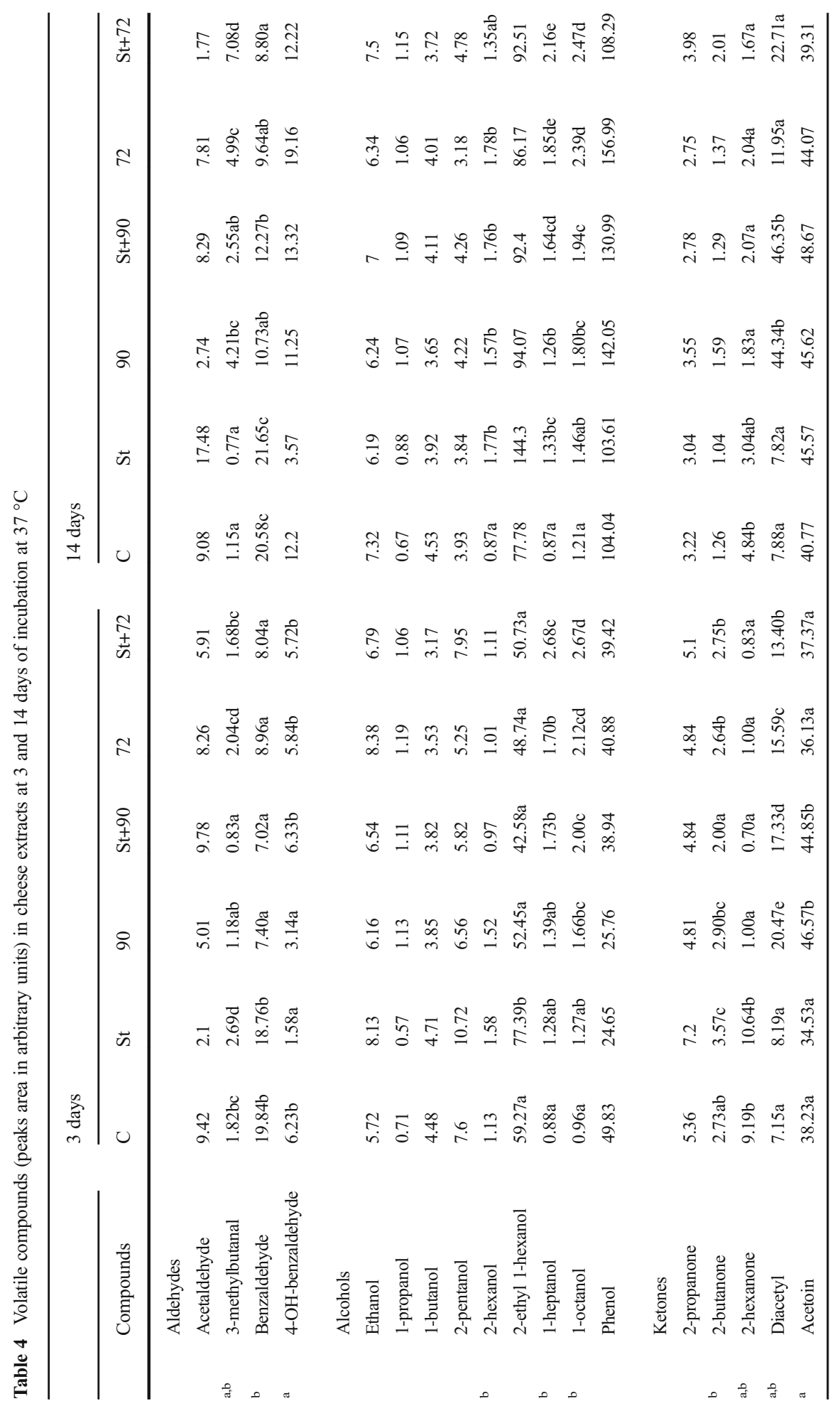




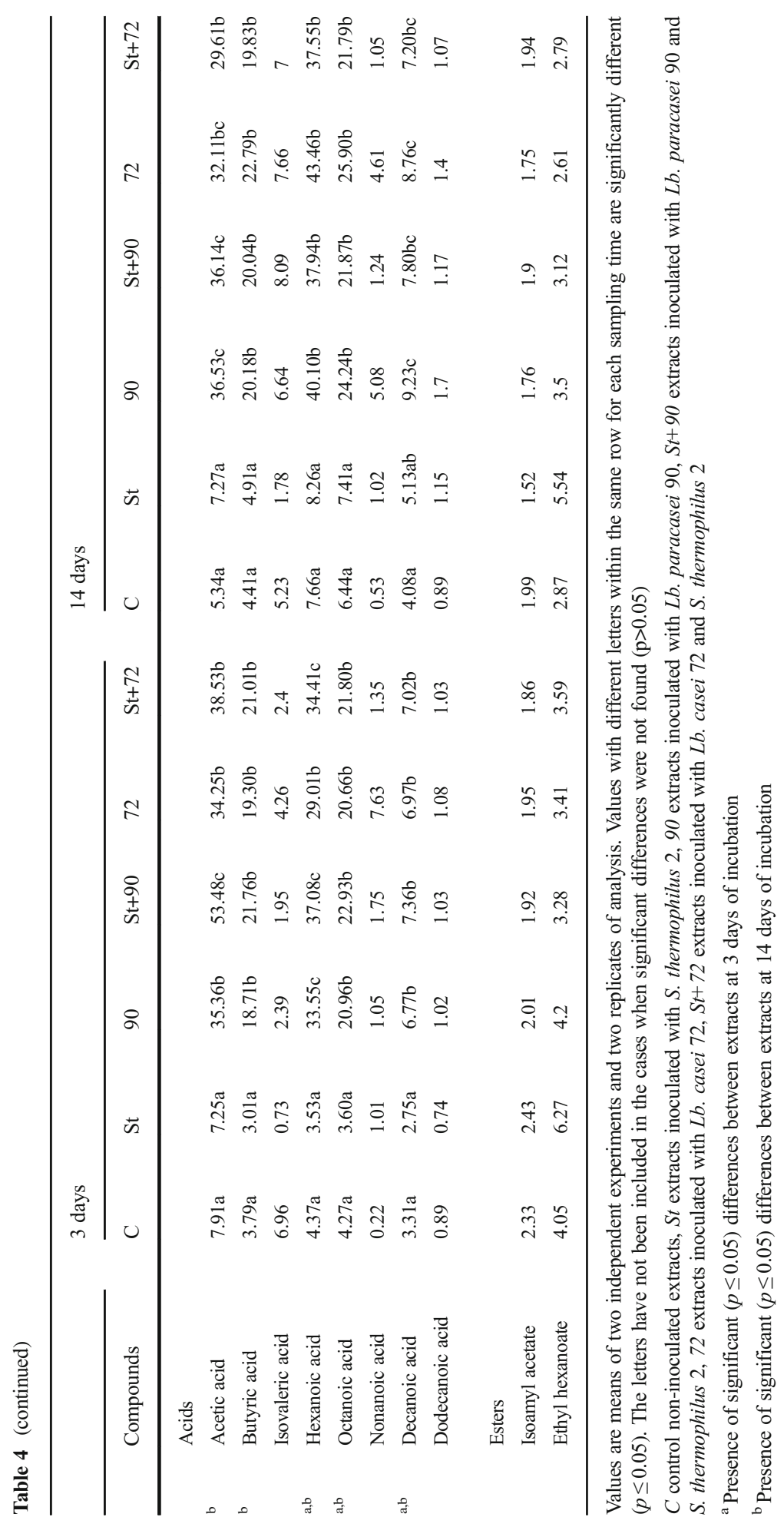


was also inoculated. In addition, 90 and St+90 extracts had much higher levels of diacetyl than did all of the other extracts after 14 days of incubation. When compared to C, 2-hexanone decreased in extracts with lactobacilli at days 3 and 14, while in St extracts, it decreased slightly only on day 14. 2-butanone levels increased in St extracts after 3 days of incubation.

Four aldehydes were found: acetaldehyde, 3-methyl butanal, benzaldehyde and 4$\mathrm{OH}$-benzaldehyde. 3-methyl butanal levels increased in St extracts compared to $\mathrm{C}$, while extracts $\mathrm{St}+90$ showed a significant decrease, both after 3 days of incubation. At 14 days, the levels of 3-methyl butanal increased in all extracts with lactobacilli in relation to $\mathrm{C}$ and $\mathrm{St}$ extracts; the values of this compound, in increasing order, were as follows: $\mathrm{C}-\mathrm{St}<\mathrm{St}+90<90-72<\mathrm{St}+72$.

Benzaldehyde levels were diminished in all extracts with lactobacilli at 3 and 14 days of incubation in relation to $\mathrm{C}$ and $\mathrm{St}$, while 4-OH-benzaldehyde levels decreased in St and 90 extracts at 3 days of incubation.

Eight acids were found in the extracts. The levels of nonanoic and dodecanoic acids were similar between extracts, while significant differences $(p \leq 0.05)$ were found for acetic, butyric, hexanoic, octanoic and decanoic, which showed increased amounts in extracts with lactobacilli. In addition, the increase of acetic and hexanoic acids (at 3 days) for extracts with the strain 90 and 72, respectively, was higher when also was present the strain of streptococci. In general, these compounds are derived from lipolysis, with the exception of acetic acid, which can be produced from several pathways. Isovaleric acid (3-methyl butanoic acid), a branched-chain fatty acid related to the catabolism of Leu, did not show significant differences $(p \geq 0.05)$ between extracts, but it diminished in St extracts in relation to the others.

The levels of the esters isoamyl acetate and ethyl hexanoate were similar in all of the extracts.

PCA was applied on the values of the 15 compounds which showed significant differences between extracts. Four PCs were extracted, which accounted $85.1 \%$ of total variance; in Fig. 4, score and loading plots for PC1 vs. PC2 are shown. Samples separated along the first PC $(51.7 \%)$, mainly according to the inoculation with lactobacilli: scores were positive for extracts 90, St+90, 72 and $\mathrm{St}+72$ and negative for $\mathrm{C}$ and St. This indicated that extracts with lactobacilli were characterised by high levels of acids, alcohols, diacetyl and acetoin and 4-OH benzaldehyde, which had high positive loadings on PC1. On the contrary, C and St extracts were characterised by 2-hexanone and benzaldehyde, variables that had high negative loading on this PC. As for PC2, samples grouped on this axe according to the lactobacilli strain inoculated (90 or 72), independently if they were as single cultures or mixed with St. Overall, samples containing the strain 90 showed higher scores on PC2 than did those with the strain 72 at the same incubation time, which was related with higher levels of acetoin, diacetyl and 4$\mathrm{OH}$ benzaldehyde. On the contrary, samples with the strain 72 were characterised with the variables with lower loading values on PC2 such as 1-heptanol, 1octanol, 3-methyl butanal and 2-butanone. There was not a clear differentiation between samples with lactobacilli and lactobacilli + streptococci.

Extracts with $\mathrm{St}$ at 3 days of incubation differentiated slightly from $\mathrm{C}$ extracts along PC2, mainly by higher levels of 2-butanone, 3-methyl butanal, 1-octanol and 1-heptanol. 
a

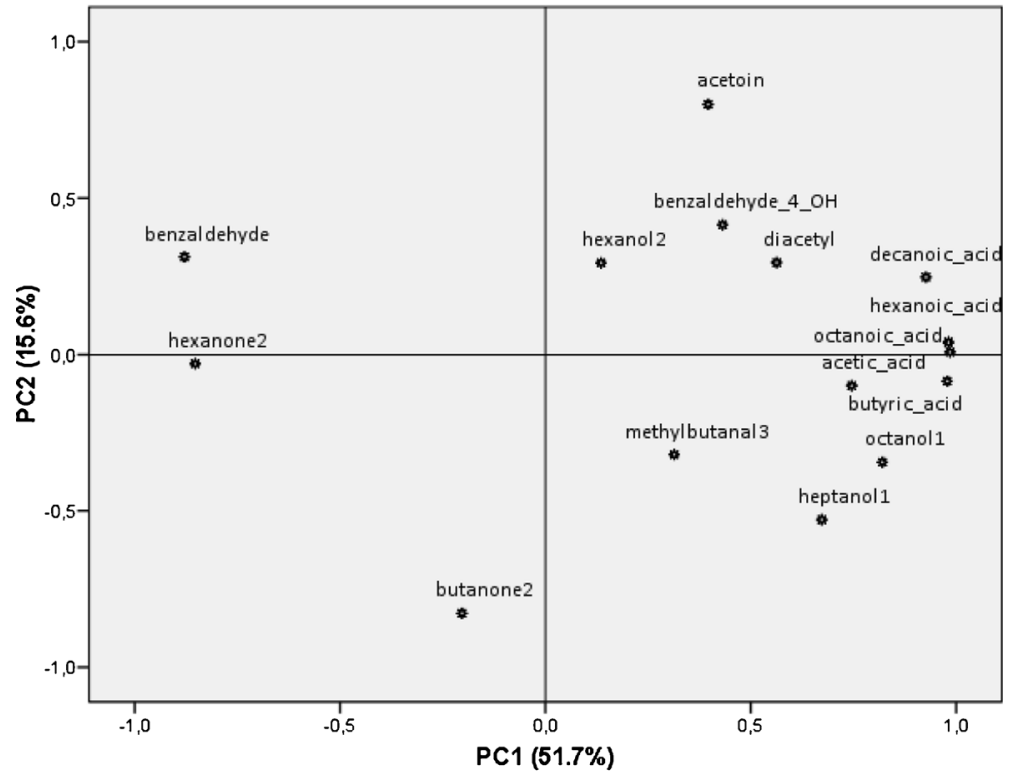

b

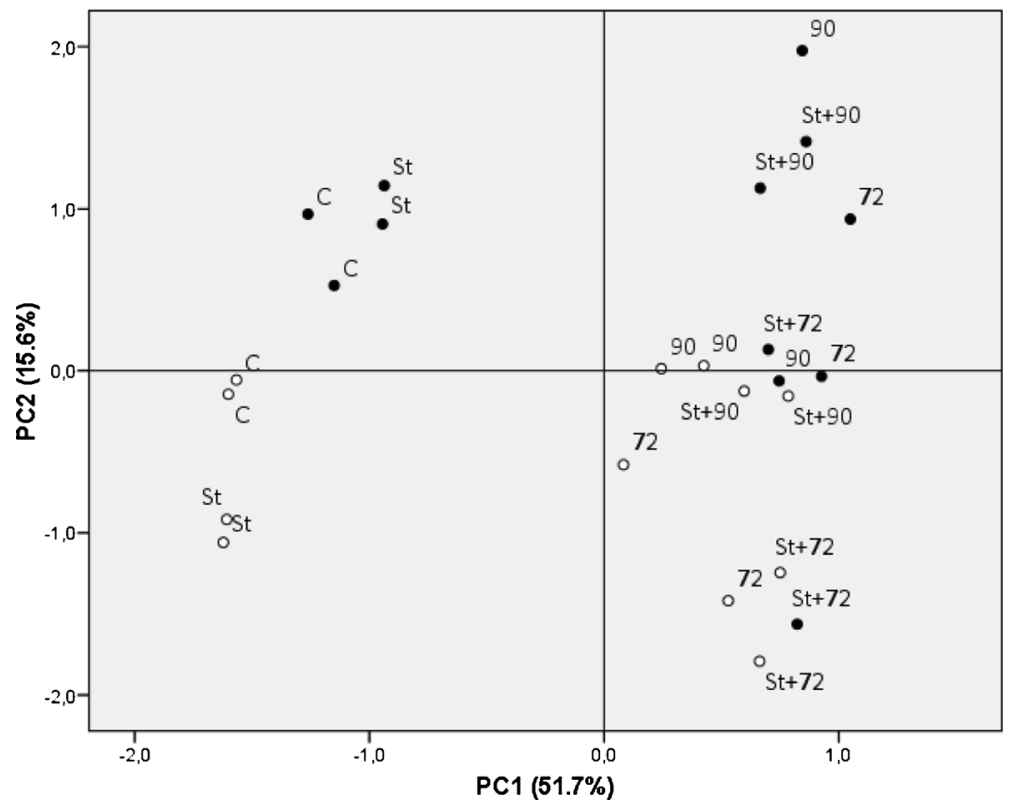

Fig. 4 Principal component analysis (PCA) of volatile compounds which presented significant differences between different extracts, at 3 (white circle) and 14 (black circle) days of incubation. a Loading plot for PC1 vs. PC2. b Score plot for PC1 vs. PC2. C: control non-inoculated extracts, St: extracts inoculated with $S$. thermophilus 2, 90: extracts inoculated with $L b$. paracasei 90, St+90: extracts inoculated with $L b$. paracasei 90 and $S$. thermophilus 2, 72: extracts inoculated with $L b$. casei 72, St+72: extracts inoculated with $L$ b. casei 72 and $S$. thermophilus 2 


\section{Discussion}

In the present work, we found that the biochemical changes leading to flavour formation in a cheese extract were mainly due to the activity of $L b$. casei 72 and $L b$. paracasei 90 . Little activity of $S$. thermophilus 2 was evidenced when the strain was assayed as a single culture, and cooperation was only observed in the production of a few compounds when this strain was mixed with the lactobacilli.

The growth and survival of both lactobacilli strains tested in the extract showed good resistance and adaptation to the environmental conditions, which is in agreement with the NSLAB origin of these strains (Briggiler-Marcó et al. 2007; Bude-Ugarte et al. 2006). On the contrary, the poor survival of $S$. thermophilus 2 reinoculated in this medium was probably due to the low tolerance of the starter to low $\mathrm{pH}$. In this sense, S. thermophilus has the ability to maintain the internal $\mathrm{pH}$ while it grows in milk at $\mathrm{pH}$ values between 6.8 and 5.2, but below this $\mathrm{pH}$, this ability is partially lost and the viability of S. thermophilus can be affected (Hutkins and Nannen 1993).

Galactose was the main carbohydrate in the extracts, which showed a concentration ten times higher than that of lactose, which was expected, as $S$. thermophilus 2 was the only starter added during cheese making. During incubation of the extract, $L b$. paracasei 90 and Lb. casei 72 fermented galactose and lactose, but S. thermophilus 2 did not cause any further changes. The ability to ferment the remaining carbohydrates in cheese is a suitable technological property for an adjunct culture to avoid the growth of spoilage microorganisms. As can be seen from our results, cheeses made with S. thermophilus 2 may benefit from the incorporation of mesophilic lactobacilli adjunct cultures for this purpose. However, overacidification is a risk that must be avoided (Crow et al. 2001). So, the effect of these lactobacilli strains on the cheese acidification should be verified before their use as adjunct cultures. However, a less pronounced overacidification is expected in cheeses, due to their buffer capacity.

It is interesting to gain insight into how amino group acceptors are produced and consumed in cheese, such as pyruvic acid and $\alpha$-ketoglutaric acid. However, as intermediate compounds, correlation to a given metabolic pathway is difficult in complex matrixes (Liu 2003). After 14 days of incubation, the two tested lactobacilli produced completely different profiles of these two compounds: while an increase in pyruvic acid characterised $L b$. paracasei 90 cultures, the extracts inoculated with $L b$. casei 72 showed an increase in $\alpha$-ketoglutaric acid levels. In general, the production of pyruvic acid surpasses consumption in all extracts with lactobacilli, but this effect was more marked for extracts with $\mathrm{Lb}$. paracasei 90 . On the contrary, S. thermophilus 2 alone was not able to increase the concentration of pyruvate in the extract when reinoculated. Conversely, $\alpha$-ketoglutaric acid is a less ubiquitous intermediate compound, as it participates in fewer metabolic pathways than pyruvate does; it is involved mainly in production via GDH activity on glutamate and consumption in transamination reactions (Gutiérrez-Méndez et al. 2008; Tanous et al. 2005; Yvon 2006). The increase in the concentration of $\alpha$-ketoglutaric acid during incubation in St extracts is in agreement with the high levels of GDH activity of $S$. thermophilus 2 (Peralta et al. 2016). On the other hand, the production of this compound in extracts with the strain 72, which had very low levels of GDH NAD-dependent activity (Peralta et al 2016), could be through the oxidative branch of the tricarboxylic acid cycle (via the citrateisocitrate pathway), in which there is an intermediate production of citric acid (Tanous 
et al. 2005). In effect, an increase of $\alpha$-ketoglutaric acid and citric acid was observed in the extracts containing this strain (72 and St+72 extracts). In St+72 extracts, no additional increase in $\alpha$-ketoglutaric acid levels attributable to cooperation during coculture was detected. On the contrary, Lb. paracasei 90 caused a decrease in $\alpha$ ketoglutaric acid levels whenever present in an extract, suggesting that there was consumption of the $\alpha$-ketoglutaric acid produced by $S$. thermophilus 2 . However, this fact did not correlate with changes in the volatilome of the extract St+90, because no cooperation of $S$. thermophilus 2 with the strain 90 was evidenced in the formation of volatile compounds derived from FAA. On the other hand, the low $\mathrm{pH}$ in extracts with lactobacilli could also have affected the GDH activity of the streptococci; in effect, the optimum pH of this enzyme is alkaline (De Angelis et al. 2010; Tanous et al. 2002; Williams et al. 2006).

The content of FAAs in extracts is the end result between its production by peptidolysis and its catabolism. Both lactobacilli strains tested showed to lead the production of FAAs during incubation of the extracts; the increase was higher for strain 72 .

In addition to increasing the total amount of FAAs, the activities of $L b$. paracase 90 and $L b$. casei 72 in the extract modified the profile of FAA, with a selective increase in the levels of AAs that are precursors of important flavour compounds, such as Leu, Glu and Asp. In this sense, Glu and Asp were minor AAs in control extracts, but they were among the most concentrated AAs in extracts inoculated with lactobacilli. In addition, Leu was the main AA in all extracts, but its concentration was much higher in extracts with lactobacilli. The amounts of Leu and Glu in extracts with strain 72 were two times higher than those in extracts with strain 90, while lower differences were found for Asp. Changes in the profiles and concentration of FAAs due to the peptidolysis may impact in the regulation of the synthesis of specific enzymes involved in their catabolism (Jensen and Ardö 2010; Pedersen et al. 2013). The fact that different types of cheeses have similar relative proportions of FAAs but very different flavours and the evidence that no flavour increase was verified when AAs were directly added into the cheese matrix showed that there is not a direct correlation between flavour and the concentrations of FAAs (McSweeney and Sousa 2000; Yvon 2006; Yvon et al. 1998). However, it is important to take into account that the different strains and species of LAB and other microbes used in cheese making may convert a similar pool of FAAs in different volatilomes according to their enzymatic abilities. In this sense, Kieronczyk et al. (2004) showed that Lb. paracasei INF15D, which has five to tenfold higher aminotransferase (AT) activity towards Asp than towards branched-chain amino acids, produced volatile compounds mainly from Asp in a cheese model. In addition, it was demonstrated that a correlation between the level of aldehydes and the concentration of the corresponding FAA precursors in different cheeses exists (Bergamini et al. 2010; Bintsis and Robinson 2004). In our work, the composition of the extract volatilome for the strain 90 correlated with the AT profile of the strain inoculated. In effect, this strain demonstrated high specificity of AT towards Asp (Peralta et al 2016) and produced a very marked increase of diacetyl and acetoin during incubation of the extracts. Although these volatile compounds can derive both from citrate and from Asp metabolism, citric acid remained constant during incubation, suggesting that Asp was the precursor. Diacetyl and acetoin have been reported to be typical flavour compounds of soft and fresh cheese varieties, characterised by a buttery note, produced mainly in 
these cheeses by citrate metabolism by citrate positive strains (McSweeney and Sousa 2000). As for $L b$. casei 72, its main influence in the production of volatile compounds derived from AAs was the increase in the levels of 3-methylbutanal, which is produced from Leu. Lb. casei 72 is characterised by a similar level of AT activity towards Met, Asp and aromatic and branched-chain AAs (Peralta et al. 2016). Among these AAs, Leu was predominant in extracts. Regarding the influence of $S$. thermophilus 2, an increase in the level of 3-methylbutanal was found after 3 days of incubation in the extract St; the AT activity of this strain was expressed mainly towards Asp, Leu and Tyr, but Leu was the main FAA in these extracts while Asp and Tyr were minor FAAs.

A concern when adjunct cultures are used is the production of acetic acid, which may impart a pungent and overly acidic taste to cheese and have a detrimental effect on flavour quality. However, this acid can be a flavour enhancer at low levels (Broadbent et al. 2011). In extracts with lactobacilli, an increase in acetic acid levels was verified. It can be derived from the metabolism of AAs, citric or lactic acids (Liu 2003).

The three strains also showed the ability to produce other volatile compounds not derived from FAA catabolism. In particular, several acids (butyric, hexanoic, octanoic and decanoic) that can be derived from lipolysis (McSweeney and Sousa 2000) increased in the extracts with Lb. paracasei 90 and Lb. casei 72, and some alcohols increased in all inoculated extracts. Helinck et al. (2004) also showed an increase in alcohols and acids by a strain of $L b$. delbrueckii subsp. lactis, but results on mesophilic lactobacilli contribution to cheese lipolysis are scarce (Hynes et al. 2003).

The influence on the production of volatile compounds by mixed cultures could be different from the individual contributions because positive or negative interactions or complementation of metabolic pathways could occur (Smit et al. 2009). In our work, changes in volatile compounds were observed mainly by the lactobacilli inoculation, while some slight variations were detected due to the joint presence of lactobacilli and streptococci. In this sense, cooperation was only observed for few compounds: 3methylbutanal in extracts with the strain 72 and 1-heptanol and acetic and hexanoic acids in extracts with any one of the lactobacilli strains. In addition, it was also observed a diminution of diacetyl due to the incorporation of streptococci in extracts with lactobacilli.

\section{Conclusions}

In this work, we showed that biochemical changes leading to flavour development in a cheese extract were led by cultures of Lb. casei 72 and Lb. paracasei 90. $S$. thermophilus 2 had an influence in the background composition of the extract, but its contribution to peptidolysis and carbohydrate fermentation during the incubation was negligible. Little evidence on $S$. thermophilus and lactobacilli cooperation on the volatile bioformation was found.

Our results also suggest that the AT profile and the peptidolytic activity of each strain influenced the volatilome of the extracts during incubation.

Both lactobacilli strains demonstrated technological properties for use as adjunct cultures in cheeses made with a starter of $S$. thermophilus to control the accumulation of galactose, accelerate peptidolysis and enhance or diversify the cheese flavour via amino acid catabolism. Lb. paracasei 90 increased the production of flavour 
compounds associated with fresh and buttery notes, and Lb. casei 72 increased peptidolysis and 3-methylbutanal.

In the present work, we studied non-growing cells of $S$. thermophilus 2. Further studies are needed to deepen the knowledge about the influence on the production of flavour compounds from amino acid of $S$. thermophilus 2, making experiences with cells in different physiological states and in other cheese models.

Acknowledgements The authors gratefully acknowledge the doctoral fellowship of Guillermo Hugo Peralta given by CONICET and the financial support given by the Agencia Nacional de Promoción Científica y Tecnológica (ANPCyT).

\section{References}

Amárita F, Requena T, Taborda G, Amigo L, Peláez C (2001) Lactobacillus casei and Lactobacillus plantarum initiate catabolism of methionine by transamination. J Appl Microbiol 90:971-978

Ayad EH, Verheul A, Engels WJ, Wouters JT, Smit G (2001) Enhanced flavour formation by combination of selected lactococci from industrial and artisanal origin with focus on completion of a metabolic pathway. $\mathrm{J}$ Appl Microbiol 90:59-67

Banks JM, Yvon M, Gripon JC, Fuente MADL, Brechany EY, Williams AG, Muir DD (2001) Enhancement of amino acid catabolism in Cheddar cheese using $\alpha$-ketoglutarate: amino acid degradation in relation to volatile compounds and aroma character. Int Dairy J 11:235-243

Bergamini CV, Wolf IV, Perotti MC, Zalazar CA (2010) Characterisation of biochemical changes during ripening in Argentinean sheep cheeses. Small Ruminant Res 94:79-89

Bintsis T, Robinson RK (2004) A study of the effects of adjunct cultures on the aroma compounds of Feta-type cheese. Food Chem 88:435-441

Briggiler-Marcó M, Capra ML, Quiberoni A, Vinderola G, Reinheimer JA, Hynes E (2007) Nonstarter Lactobacillus strains as adjunct cultures for cheese making: in vitro characterization and performance in two model cheeses. J Dairy Sci 90:4532-4542

Broadbent JR, Budinich MF, Steele JL (2011) Non-starter lactic acid bacteria. In: Fuquay JW, Fox PF, McSweeney PLH (eds) Encyclopedia of dairy science. Academic Press, Oxford, pp 639-644

Bude-Ugarte M, Guglielmotti D, Giraffa G, Reinheimer J, Hynes E (2006) Nonstarter lactobacilli isolated from soft and semihard Argentinean cheeses: genetic characterization and resistance to biological barriers. J Food Protect 69:2983-2991

Crow V, Curry B, Hayes M (2001) The ecology of non-starter lactic acid bacteria (NSLAB) and their use as adjuncts in New Zealand Cheddar. Int Dairy J 11:275-283

De Angelis M, Calasso M, Di Cagno R, Siragusa S, Minervini F, Gobbetti M (2010) NADP-glutamate dehydrogenase activity in nonstarter lactic acid bacteria: effects of temperature, $\mathrm{pH} \& \mathrm{NaCl}$ on enzyme activity and expression. J Appl Microbiol 109:1763-1774

Di Cagno R, De Pasquale I, De Angelis M, Buchin S, Calasso M, Fox PF, Gobbetti M (2011) Manufacture of Italian Caciotta-type cheeses with adjunct and attenuated adjuncts of selected non-starter lactobacilli. Int Dairy J 21:254-260

Ganesan B, Stuart MR, Weimer BC (2007) Carbohydrate starvation causes a metabolically active but nonculturable state in Lactococcus lactis. Appl Environ Microbiol 73:2498-2512

Gutiérrez-Méndez N, Valenzuela-Soto E, González-Córdova AF, Vallejo-Cordoba B (2008) $\alpha$-Ketoglutarate biosynthesis in wild and industrial strains of Lactococcus lactis. Lett Appl Microbiol 47:202-207

Helinck S, Le Bars D, Moreau D, Yvon M (2004) Ability of thermophilic lactic acid bacteria to produce aroma compounds from amino acids. Appl Environ Microbiol 70:3855-3861

Hutkins RW, Nannen NL (1993) pH homeostasis in lactic acid bacteria. J Dairy Sci 76:2354-2365

Hynes E, Bach C, Lamberet G, Ogier J-C, Son O, Delacroix-Buchet A (2003) Contribution of starter lactococci and adjunct lactobacilli to proteolysis, volatile profiles and sensory characteristics of washed-curd cheese. Lait 83:31-43

Jensen M, Ardö Y (2010) Variation in aminopeptidase and aminotransferase activities of six cheese related Lactobacillus helveticus strains. Int Dairy J 20:149-155 
Kieronczyk A, Skeie S, Langsrud T, Yvon M (2003) Cooperation between Lactococcus lactis and nonstarter lactobacilli in the formation of cheese aroma from amino acids. Appl Environ Microbiol 69:734-739

Kieronczyk A, Skeie S, Langsrud T, Le Bars D, Yvon M (2004) The nature of aroma compounds produced in a cheese model by glutamate dehydrogenase positive Lactobacillus INF15D depends on its relative aminotransferase activities towards the different amino acids. Int Dairy J 14:227-235

Liu S-Q (2003) Practical implications of lactate and pyruvate metabolism by lactic acid bacteria in food and beverage fermentations. Int J Food Microbiol 83:115-131

McSweeney PLH, Sousa MJ (2000) Biochemical pathways for the production of flavour compounds in cheese during ripening. Lait 80:293-324

Milesi MM, Vinderola G, Sabbag N, Meinardi CA, Hynes E (2009) Influence on cheese proteolysis and sensory characteristics of non-starter lactobacilli strains with probiotic potential. Food Res Int 42:1186-1196

Milesi MM, Wolf IV, Bergamini CV, Hynes ER (2010) Two strains of nonstarter lactobacilli increased the production of flavor compounds in soft cheeses. J Dairy Sci 93:5020-5031

Pedersen TB, Ristagno D, McSweeney PLH, Vogensen FK, Ardö Y (2013) Potential impact on cheese flavour of heterofermentative bacteria from starter cultures. Int Dairy J 33:112-119

Peralta GH, Wolf IV, Bergamini CV, Perotti MC, Hynes ER (2014) Evaluation of volatile compounds produced by Lactobacillus paracasei 190 in a hard-cooked cheese model using solid-phase microextraction. Dairy Sci Technol 94:73-81

Peralta GH, Bergamini CV, Hynes ER (2016) Aminotransferase and glutamate dehydrogenase activities in lactobacilli and streptococci. Braz J Microbiol. doi:10.1016/j.bjm.2016.04.005

Porcellato D, Johnson ME, Houck K, Skeie SB, Mills DA, Kalanetra KM, Steele JL (2015) Potential of Lactobacillus curvatus LFC1 to produce slits in Cheddar cheese. Food Microbiol 49:65-73

Smit BA, Engels WJM, Smit G (2009) Branched chain aldehydes: production and breakdown pathways and relevance for flavour in foods. Appl Microbiol Biotechnol 81:987-999

Steele J, Broadbent J, Kok J (2013) Perspectives on the contribution of lactic acid bacteria to cheese flavor development. Curr Opin Biotechnol 24:135-141

Tanous C, Kieronczyk A, Helinck S, Chambellon E, Yvon M (2002) Glutamate dehydrogenase activity: a major criterion for the selection of flavour-producing lactic acid bacteria strains. Antonie Van Leeuwenhoek 82:271-278

Tanous C, Gori A, Rijnen L, Chambellon E, Yvon M (2005) Pathways for $\alpha$-ketoglutarate formation by Lactococcus lactis and their role in amino acid catabolism. Int Dairy J 15:759-770

Thage BV, Broe ML, Petersen MH, Petersen MA, Bennedsen M, Ardö Y (2005) Aroma development in semihard reduced-fat cheese inoculated with Lactobacillus paracasei strains with different aminotransferase profiles. Int Dairy J 15:795-805

Wang W, Zhang L, Li Y (2012) Production of volatile compounds in reconstituted milk reduced-fat cheese and the physicochemical properties as affected by exopolysaccharide-producing strain. Molecules 17:14393-14408

Williams AG, Withers SE, Brechany EY, Banks JM (2006) Glutamate dehydrogenase activity in lactobacilli and the use of glutamate dehydrogenase-producing adjunct Lactobacillus spp. cultures in the manufacture of cheddar cheese. J Appl Microbiol 101:1062-1075

Yvon M (2006) Key enzymes for flavor formation by lactic acid bacteria. Aust J Dairy Technol 61:16-24

Yvon M, Berthelot S, Gripon JC (1998) Adding $\alpha$-ketoglutarate to semi-hard cheese curd highly enhances the conversion of amino acids to aroma compounds. Int Dairy J 8:889-898 